\title{
Interactive comment on "Simultaneous retrieval of water vapour and temperature profiles and cirrus clouds properties from measurements of far infrared spectral radiance over the Antarctic Plateau" by Gianluca Di Natale et al.
}

Gianluca Di Natale et al.

luca.palchetti@ino.it

Received and published: 9 November 2016

\section{Author response to Referee \#3}

We thank the Reviewer for the comments that we believe have allowed to improve our paper.

Below we address point-by-point all the questions raised by the review, describing the changes that we have done in the manuscript. We make reference to page $(P)$ and line $(\mathrm{L})$ of the updated manuscript (file $\mathrm{AC} 1$-supplement). 
Major Comment 1. Many of the assumptions made are not suitably justified and the associated uncertainty analyses are inadequate to appropriately characterize the performance of the algorithm. For example, the statement "The strongest assumption, which is considered sufficiently reliable, is the approximation of a single uniform layer" is not justified in any way despite the availability of statistics from active sensors. In addition, the uncertainty owing to the assumption of hexagonal columns is not assessed. Also, the potential influence of the presence of super-cooled liquid is not addressed. In fact, other than measurement noise, it appears that only errors in the $\mathrm{CO} 2$ profile are actually considered in defining the observation error covariance matrix (Eqn. 10) but this must also account for errors in forward model assumptions.

Reply. The assumption of single uniform layer is typically used to describe the radiative effect of ice cirrus clouds since these clouds are optically thin and the internal stratification shows a small effect on the radiative transfer, see also e.g Turner et al. 2003,2005 , where the same approximation is used. Furthermore this assumption has been verified in our specific cases where the optical depth $(\tau)$ of cirrus clouds is less than 1.2. In this condition the radiance difference between the simulated spectra using a single layer of $1 \mathrm{~km}$ or a vertical stratification of $100 \mathrm{~m}$ is within the measurement uncertainty of REFIR-PAD. Figure 1 for example, shows the two simulated spectra performed for a single uniform layer of $1 \mathrm{~km}$ (black line) and using a stratification of $100 \mathrm{~m}$ (red line). The difference (green curve) is negligible with respect to the measurement uncertainty (blue lines).

We have rewritten the sentences at P3 L8-13 to be clearer and to include the above cited references. The text has been modified to:

"The assumption of single uniform layer is typically used to describe the radiative effect of ice cirrus clouds since these clouds are optically thin (Mahesh et al. 2001b) and the internal stratification shows a small effect on the radiative transfer, see also e.g Turner
AMTD

Interactive comment
Printer-friendly version

Discussion paper

2 
et al. (2003) and Turner (2005) where the same approximation is used. Furthermore this assumption has been verified in our specific cases, where $\tau$ of cirrus clouds is less than 1.2, finding that the effect of considering the stratification produces a difference that is negligible with respect to the measurement noise."

The choice of hexagonal columns is motivated by the current knowledge of the typical habit composition in polar cirrus clouds. This choice has been addressed in more details by adding at P3 L14-20 the following text:

"Currently there is very little information about the statistical distribution of shapes of ice particles in polar regions, e.g. Mahesh et al. (2001b) for Antarctica and Turner et al. (2003) for the Arctic show the predominance of column particles. In particular Turner et al. (2003) showed that the typical polar habits are essentially composed by hexagonal columns with a minor fraction of droxtal for small particles. Furthermore also the single scattering models developed are very few (Fu and Liou, 1993; Fu et al., 1998; Yang at al., 2005) and not validated over the whole spectral range because of the lack of measurements in the FIR. The model developed by Fu et al. (1998) has been chosen in this analysis because it describes well clouds composed of a mixture of hexagonal columns where the shape approximates the droxtal for small size particles with aspect ratio near to $1 . "$

Furthermore we have also compared simulations performed in the case of $100 \%$ of hexagonal columns and with a fraction of $50 \%$ hexagonal columns and $50 \%$ droxtal. The results shown in Fig. 2 demonstrate that the modelling differences are very small and well within the measurement error.

The presence of mixed phase clouds with super-cooled liquid can be well identified by the lidar depolarization signal. Actually the algorithm is also able to perform the retrieval of ice fraction and effective liquid droplets but, as said in the text, we have limited the analysis to thin ice clouds. In such a way the retrieval configuration is a bit simpler and the discussion about the performance of the simultaneous retrieval of also 
the atmospheric state can be more straightforward. For this reason we have analysed the measurements in coincidence with radiosoundings and only one example of the results for each season is reported in the paper.

Finally the VCM, which we have used in the retrieval (Eqn. 10), takes into account only the predominant source of error in the forward model, comparable with the measurement errors in the spectral band covered by REFIR-PAD, i.e. the error due to the uncertainty on the $\mathrm{CO}_{2}$ climatological profile. The following clarification has been added to the text at P6 L26-27: " $\sigma_{F}$ is the forward model error due to the uncertainties in the non fitted species and the assumption done in the description of the cloud properties." , and further at at P7 L1-5: "The forward model error $\sigma_{F}$ is dominated by the uncertainty on the $\mathrm{CO}_{2}$ climatological profile, which is obtained by means of the standard deviation $\sigma_{\mathrm{CO}_{2}}$ of the $\mathrm{CO}_{2}$ profile (Remedios et al. 2007) and the derivative of the forward model with respect to the $\mathrm{CO}_{2}$ volume mixing ratio. The other non fitted atmospheric species, the single layer approximation and the choice of hexagonal columns for the cloud description have a negligible effect on the VCM compared to the measurement noise."

This sentences appear just after Eqn. 10.

The reference Palchetti and Lastrucci, 2001 has been modified to: Bianchini and Palchetti, 2008.

Major Comment 2. The values of the a priori errors are never actually stated in the paper - they are merely stated to be "large enough to not be serious constraints". In addition, it is assumed that effective diameter and ice water path are uncorrelated and cloud and the atmospheric properties are assumed uncorrelated. In reality observations show that all of these quantities are strongly correlated, larger particles tend to be observed when ice water paths are large and cloud formation is strongly related to relative humidity (supersaturation). Why aren't these correlations treated in a similar

\section{AMTD}

Interactive comment
Printer-friendly version

Discussion paper 
manner to the temperature and humidity profiles on page 10? Curiously, one of the key results of the paper actually involves defining relationships between cloud optical depth and temperature and effective diameter and IWC yet these correlations are not modeled in the algorithm. In addition, the conclusions actually state "This work has shown the capability to perform a simultaneous retrieval of the atmospheric state and the cloud parameters taking into account the possible correlations between the clouds and the atmosphere" yet it isdiz clear from the algorithm description that these correlations are NOT explicitly treated.

Reply. We have modified the text by adding the a priori errors that we have used in the analysis. With reference Eq. (10), the text at P6 L26 - P7 L5 has been modified to: $" \sigma_{F}$ is the forward model error due to the uncertainties in the non fitted species and the assumption done in the description of the cloud properties. The term composed of NESR and $\sigma_{F}$ denotes the uncorrelated statistical error, whereas the term of products $\epsilon_{i} \cdot \epsilon_{j}$ represents the correlated error component given by the calibration uncertainty with correlation equal to 1 , as derived from the Planck law of emission. The forward model error $\sigma_{F}$ is dominated by the uncertainty on the $\mathrm{CO}_{2}$ climatological profile, which is obtained by means of the standard deviation $\sigma_{C O_{2}}$ of the $\mathrm{CO}_{2}$ profile (Remedios et al. 2007) and the derivative of the forward model with respect to the $\mathrm{CO}_{2}$ volume mixing ratio. The other non fitted atmospheric species, the single layer approximation and the choice of hexagonal columns for the cloud description have a negligible effect on the VCM compared to the measurement noise."

Concerning the parameter correlations, we agree with the Referee that De, IWP, and the atmospheric state are strongly correlated. In the case of the atmospheric temperature and humidity vertical profiles, the correlation is taken into account in the a priori VCM, see Eqn. 13, because we have performed a climatology of these parameters with the available radiosoundings. However only a few measurements exists for the Antarctic plateau on cirrus cloud parameters to perform a rigorous statistical analysis of the correlation between De and IWP, and between these parameters and the atmo-

Interactive comment 
spheric state. See for example Mahesh et al. 2001 (added to the revised manuscript) where the analysis was performed with Mie approximation. Therefore we have chosen not to constrain the retrieval with the a priori correlations. In such a way, the results of the simultaneous fitting of the cloud parameters and the atmospheric state can allow us to put in evidence the existing correlation among them. Then, as a first qualitative evidence for a selected set of measurements in coincidence with the radiosoundings and in presence of cirrus clouds, we have compared the retrieved atmospheric state with radiosondings and shown that the resulting cloud parameters distributions shows a behaviour that is similar to analogous statistical distributions obtained in the Arctic and on the Antarctic coast. The text has been changed in order to take into account the above clarification. In particular, the word "correlation" has been changed to "spectral interference", whenever appropriate, and used only for the statistical correlation between parameters. Thus in the Abstract the sentence at P1 L2-4 has been changed to: "A simultaneous retrieval of cloud and atmospheric parameters from infrared wideband spectra will allow the disentanglement of the spectral interference between these variables."

The statement has been modified at P1 L19-21 to: "Finally, the retrieved cloud parameters put in evidence correlation laws between cloud temperature and optical depth, and between effective particle diameter and ice water content. These correlations are similar to the statistical correlations measured on the Antarctic coast at Dumond D'Urville and in the Arctic region."

The sentence has been changed at P1 L14-16 to: "A climatology of the vertical profiles of water vapour and temperature has been performed by using the daily radiosounding available at the Station at 12 UTC. The climatology has been used to build an a priori profile correlation to constrain the fitting procedure."

The sentence has been changed at P7 L12-16 to: "Since only a few measurements of cirrus cloud parameters in the Antarctic plateau are available, to perform a rigorous statistical analysis of the correlation between $D_{e}$ and IWP and between these parame-

Interactive comment 
ters and the atmospheric state, we have chosen not to constrain the retrieval with these a priori correlations. Therefore the off-diagonal elements of $S_{a}$ and $S_{c l d}$ are set to be equal to 0 . In this way, the results of the simultaneous fitting of the cloud parameters and the atmospheric state will put into evidence the existing correlation between these variables."

Interactive

In Section 4 (now Section 5), the following sentence has been added at P11 L28-30: "In order to have a first qualitative evaluation of the cloud parameter retrieval performances, we have compared the retrieved values distributions with the corresponding statistical distributions measured on the Antarctic coast at sea level at the Dumont D'Urville Station and in the Arctic."

Finally, the conclusive sentences have been changed at P13 L3-9 to: "The fitting procedure allows to obtain a good agreement between measurements and simulations, with the residual differences generally falling within measurement noise over the whole relevant spectral range, including the FIR. The atmospheric retrieved profiles of water vapour and temperature follow the available simultaneous radiosoundings, whereas the retrieved cirrus cloud parameters follow analogous statistical distributions available for polar regions. This work has shown the capability to perform a simultaneous retrieval of the atmospheric state and of the cloud parameters taking into account the whole spectral range in which their infrared emission is relevant, allowing in such a way to disentangle the spectral interference between the variables."

Major Comment 3. While it is good that the authors consider the information content of the measurements, the analysis presented in Section 3 lacks sufficient rigor to be informative. First the inadequate characterization of uncertainties noted in (1) and (2) above call the findings into question. In addition, no cloud parameters are actually considered in the analysis limiting their value. In order to adequately characterize the information content of the FIR measurements used in the retrieval, the error covariance matrices must include forward model errors and correlations between cloud and

Printer-friendly version

Discussion paper 
atmospheric properties must be included.

Reply. The questions raised by this comment have been addressed in the previous reply to Major Comment 2. We think that the text modifications have improved the description of the analysis presented in Section 3. Concerning the exploitation of the under-explored FIR region, we agree with the Referee, we have not quantitatively addressed in this paper the improved information content supplied by the FIR compared to the commonly-used mid-IR region. The better sensitivity of FIR spectra to cirrus cloud parameters is shown qualitatively by Fig. 1 of the paper. Moreover other papers reports about the improvement in the cloud characterisation using the FIR region. Our paper is focused on the description of the simultaneous retrieval of the atmospheric state and the cloud parameters exploiting the spectral signatures present in full band of the infrared emission, including the mid-IR and the under-explored FIR, and to disentangle the competing effects associated with them. With the exception of the humidity and temperature profiles, that we characterise in the a priori VCM, no information content analysis has been considered in the paper. We have added more information and references to the FIR exploitation at P5 L7-11.

Major Comment 4. The validation of the approach is incomplete and not convincing. Simply noting that residuals are small (page 14) does not provide a measure of the quality of the retrievals. It merely demonstrates that the retrieval has enough degrees of freedom to sufficiently fit the observations. This can always be accomplished in under-constrained problems. A limited number of direct comparisons against radiosondes are presented in Fig. 8 but the paper lacks any quantitative statistical analysis of the accuracy of the retrievals. In addition, no error bars are presented on the retrieved quantities so it is impossible to know whether agreement is achieved within the anticipated retrieval error. In some cases differences between retrieved profiles and soundings exceeds $10 \mathrm{~K}$ - is this really "very good agreement"? Finally, the comment "The comparison between the retrieved parameters and statistical correlation laws shows a
AMTD

Interactive

comment
Printer-friendly version

Discussion paper 
very good agreement" on page 16 does not rise to the level of evaluating the retrieval performance. On a related note, what exactly is meant by the statement "The comparAMTD ison of results with radiosoundings demonstrates that the retrieved atmospheric state is not disturbed by the clouds presence" in the abstract?

Reply. We agree that in under-constrained problems small residuals do not provide a measurement of the quality of the retrievals when the number of the fitted parameters exceeds the degree of freedom of the system. However, we have performed a SVD analysis, shown in the text, that has allowed to select the proper number of independent fitting variables. In this case the small residual and the general agreement with radiosoundings for humidity and temperature profiles should allow to conclude that a sufficiently good quality of the retrieval approach is achieved. Concerning the cloud parameters, to our knowledge no measurements exists for the Antarctic plateau to be used for comparison. We have only shown that the cirrus cloud retrieval has sufficient accuracy to infer possible parameter correlations.

In the paper we have chosen to show the comparison with radiosoundings only in a few cases that are representative of the results for different conditions during the year, i.e. one case per season. All the analysed cases show similar results. We have modified Fig. 8 (now Fig. 9) in order to add more fitted profiles for each single case, using the 2-3 measurements in better temporal coincidence with the sounding.

Since the comparison of a high vertical resolution profile like the radiosounding and the very rough interpolation given by the fit can only be qualitative, we added a figure (fig. 10 ) in which we compare two physical parameters (columnar water vapour - PWV and the average temperature of the first $50 \mathrm{~m}$ ) that can be obtained both from sounding and fitting (error bars included).

The difference between the fitted profiles and the radiosoundings are shown in the relevant portion of the altitude range, where the measurement is sensitive, that is between 4 (instrument altitude) to $5000 \mathrm{~m}$. Below about $10 \mathrm{~m}$, near the shelter where

Interactive comment 
the instrument is installed, the comparison for the temperature is not reliable because of the presence of the (warm) shelter itself. Above $5000 \mathrm{~m}$ near the tropopause the measurement is not sensitive as it was shown by the SVD analysis (see Figure 6, now Fig. 5) . This concept was shortly described at P11 L3-4. We have modified the text at P10 L30 - P11 L4.to describe better this limitations:

"the lowermost fitted temperature point is not shown in figure for the temperature due to several biases affecting its value:

1. the strong atmospheric variability occurring in the boundary layer;

2. the fact that the radiosonde is launched at about $500 \mathrm{~m}$ from the shelter where REFIR-PAD is located;

3. the presence of a very strong gradient in the first 3 meters of line of sight, that include the transition between the inside of the shelter, and the outside environment.

On the other side, above $5000 \mathrm{~m}$, near the tropopause level, the downwelling spectral radiance has a negligible sensitivity to atmospheric water vapor and temperature, as shown by the SVD analysis (see Fig. 7)."

Finally, Figure 8 (now Fig.9) has been updated reducing the vertical scale to $10-5000$ $\mathrm{m}$.

Finally, the last statement of Sect. 5 has been modified to: "The results of Fig. 12 show that the retrieval accuracy allows to infer distribution laws for the retrieved cloud parameters that are compatible with analogous statistical distributions. A multi-year analysis over the full dataset of our measurements is under study in order to better quantify these distribution laws."

The statement in the abstract, cited in the Referee comment, means that the simultaneous retrieval, using the full spectral band of the IR emission, of the atmospheric 
state and the cloud properties allow to disentangle the different effects that hit the same spectral regions. The statement has been modified to: "In most of the cases, the retrieved humidity and temperature profiles show good agreement with the radiosoundings, demonstrating that the simultaneous retrieval of the atmospheric state is not biased by the presence of cirrus clouds."

Interactive

comment

Major Comment 5. Finally, the reference to energy balance, cloud, and ice particle literature in the introduction is far too narrow and lacks any mention of many important recent papers on the subject. In addition, some literature specific to Antarctic clouds should be added.

Reply. The following references have been added in the Introduction:

- Baum B.A., Heymsfield A.J., Yang P. and Bedka S.T.: Bulk scattering properties for the remote sensing of ice clouds. Part I: Microphysical data and models, Journal of Applied Meteorology, 44(12), 1885-1895, 2005.

- Baum B.A., Yang P., Heymsfield A.J., Platnick S., King M.D., Hu Y. and Bedka S.T.: Bulk scattering properties for the remote sensing of ice clouds. Part II: Narrowband models, Journal of Applied Meteorology, 44(12), 1896-1911, 2005.

- Baran A. J., Hill P., Furtado K., Field P., Manners J., A coupled cloud physics-radiation parameterization of the bulk optical properties of cirrus and its impact on the Met Office Unified Model Global Atmosphere 5.0 configuration J. Climate, 27 (20), 7725-7752, 2014.

- Barton N. P., Klein S. A., and Boyle J. S.: On the Contribution of Longwave Radiation to Global Climate Model Biases in Arctic Lower Tropospheric Stability. J. Climate, 27, 7250-7269, 2014, doi: 10.1175/JCLI-D-14-00126.1

- Bromwich D. H., Nicolas J. P., Hines K. M., Kay J. E., Key E. L., Lazzara M. A., Lubin D., McFarquhar G. M., Gorodetskaya I. V., Grosvenor D. P., Lachlan-Cope T. and Van 
Lipzig N. P. M.: Tropospheric clouds in Antarctica, Rev. Geophys., 50(1), 2012.

- Choi, Y.-S., C.-H. Ho, S.-W. Kim, and R. S. Lindzen: Observational diagnosis of cloud AMTD phase in the winter antarctic atmosphere for parameterizations in climate models. Adv. Atmos. Sci., 27(6), 1233-1245, 2010, doi: 10.1007/s00376-010-9175-3.

- Cox, C.V., Harries J.E., Taylor J.P., Green P.D., Baran A.J., Pickering J.C., Last A.E., Murray J.E., Measurement and simulation of mid-and far-infrared spectra in the presence of cirrus, Quarterly Journal of the Royal Meteorological Society, 136 (648), 718739, 2010.

- Cox, C. J., Turner, D. D., Rowe, P. M., Shupe, M. D., Walden, V. P.: Cloud microphysical properties retrieved from downwelling infrared radiance measurements made at Eureka, Nunavut, Canada (2006-09). Journal of Applied Meteorology and Climatology, 53(3), 772-791, 2014.

- Cox, C. J., Walden V. P., Rowe P. M., Shupe M. D., Humidity trends imply increased sensitivity to clouds in a warming Arctic, Nature Communication, 6, 2015.

- Lubin, D., Chen B., Bromwich D. H., Somerville R. C. J., Lee W. H., and. Hines K. M.: The impact of Antarctic cloud radiative properties on a GCM climate simulation, J. Clim., 11(3), 447-462, doi:10.1175/1520-0442(1998)011<0447:TIOACR>2.0.CO;2, 1998.

- Lubin D., Kahn B. H., Lazzara M. A., Rowe P. and Walden V. P.: Variability in AIRSretrieved cloud amount and thermodynamic phase over west versus east Antarctica influenced by the SAM, Geophys. Res. Lett., 42(4), 1259-1267, 2015.

- Maestri T., Rizzi R., Smith J. A., Spectral infrared analysis of a cirrus cloud based on Airborne Research Interferometer Evaluation System (ARIES) measurements, Journal of Geophysical Research: Atmospheres, 110, (D6), 2005.

-Mahesh A., Campbell J. R., Spinhirne J. D.: Multi-year measurements of cloud base heights at South Pole by lidar, Geo. Res. Lett., 32, L09812, doi:10.1029/

Interactive comment

Printer-friendly version

Discussion paper

C12 
- Mahesh A., Walden V. P., Warren S. G.: Ground-Based Infrared Remote AMTD Sensing of Cloud Properties over the Antarctic Plateau. Part I: Cloud-Base Heights. J. Appl. Meteor., 40, 1265-1278, 2001, doi: 10.1175/15200450(2001)040<1265:GBIRSO>2.0.CO;2.

- Mahesh A., Walden V. P., Warren S. G.: Ground-Based Infrared Remote Sensing of Cloud Properties over the Antarctic Plateau. Part II: Cloud Optical Depths and Particle Sizes. J. Appl. Meteor., 40, 1279-1294, 2001, doi: 10.1175/15200450(2001)040<1279:GBIRSO>2.0.CO;2.

- Maestri, T., and Holz, R. E.: Retrieval of cloud optical properties from multiple infrared hyperspectral measurements: A methodology based on a line-by-line multiplescattering code, IEEE Transactions on Geoscience and Remote Sensing, 47(8), 24132426, 2009.

- Pithan, F., Medeiros, B., Mauritsen T.: Mixed-phase clouds cause climate model biases in Arctic wintertime temperature inversions, Clim Dyn., 43, 289, 2014, doi:10.1007/s00382-013-1964-9

- Sassen, K., Wang, Z., Liu, D. Global distribution of cirrus clouds from CloudSat/CloudAerosol lidar and infrared pathfinder satellite observations (CALIPSO) measurements, Journal of Geophysical Research - Atmospheres, 113(D8), 2008.

- Scott, R. C., and D. Lubin: Unique manifestations of mixed-phase cloud microphysics over Ross Island and the Ross Ice Shelf, Antarctica, Geophys. Res. Lett., 43, 29362945, 2015, doi:10.1002/2015GL067246

- Stocker, T. F., et al. (2013). IPCC, 2013: climate change 2013: the physical science basis. Contribution of working group I to the fifth assessment report of the intergovernmental panel on climate change, Cambridge University Press.

- Wylie D. P. and Menzel W. P.: Eight Years of High Cloud Statistics Using HIRS, J. 
Climate, 12(1), 170-184, 1999.

Interactive comment on Atmos. Meas. Tech. Discuss., doi:10.5194/amt-2016-181, 2016.
AMTD

Interactive comment 
AMTD

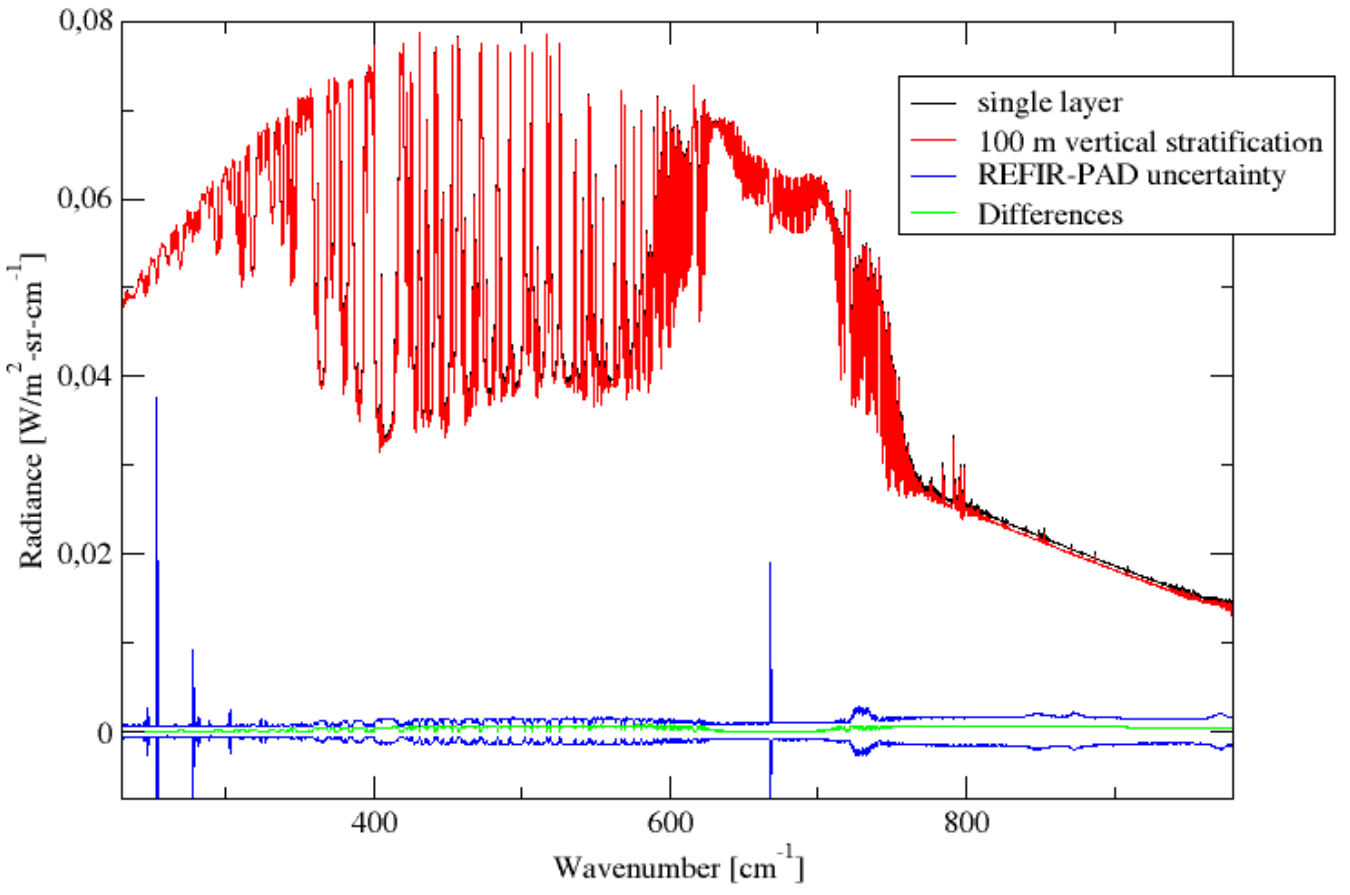

Interactive comment

Fig. 1. Simulation of the downward spectral radiance of a cirrus cloud with optical depth $=1.2$, thickness $=1 \mathrm{~km}$ and located at $800 \mathrm{~m}$ above the ground. 
AMTD

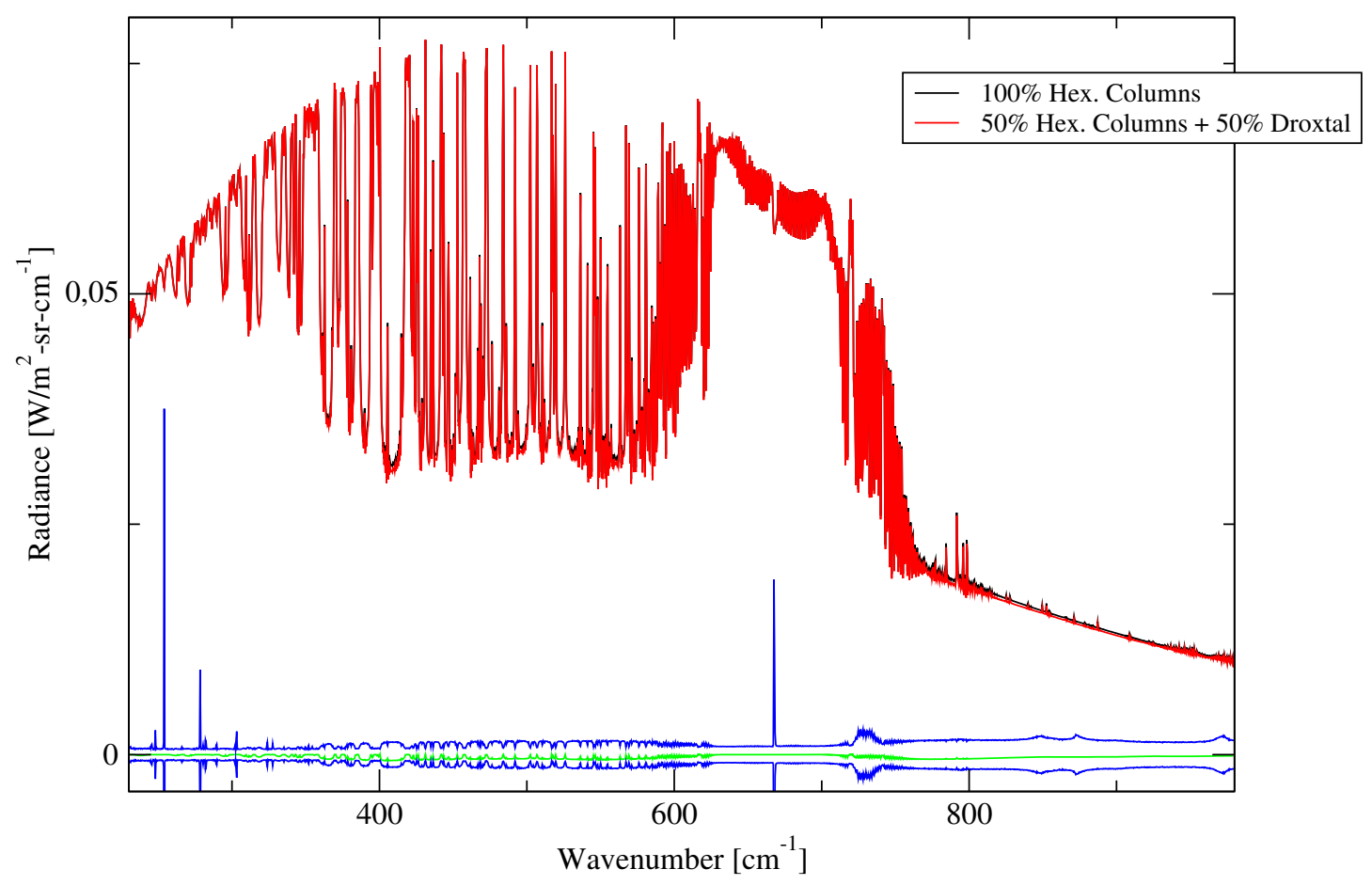

\section{Interactive comment}

Fig. 2. Simulations performed in the case of $100 \%$ of hexagonal columns (black) and with a fraction of $50 \%$ hexagonal columns and $50 \%$ droxtal (red). 